\title{
Maturity-onset diabetes of the young (MODY): how many cases are we missing?
}

\author{
B. M. Shields • S. Hicks • M. H. Shepherd • \\ K. Colclough • A. T. Hattersley $\cdot$ S. Ellard
}

Received: 27 January 2010 / Accepted: 26 April 2010/Published online: 25 May 2010

(C) Springer-Verlag 2010

\begin{abstract}
Aims/hypothesis Maturity-onset diabetes of the young is frequently misdiagnosed as type 1 or type 2 diabetes. A correct diagnosis of MODY is important for determining treatment, but can only be confirmed by molecular genetic testing. We aimed to compare the regional distribution of confirmed MODY cases in the UK and to estimate the minimum prevalence.

Methods UK referrals for genetic testing in 2,072 probands and 1,280 relatives between 1996 and 2009 were examined by region, country and test result. Referral rate and prevalence were calculated using UK Census 2001 figures. Results MODY was confirmed in 1,177 (35\%) patients, with $H N F 1 A(52 \%)$ and GCK mutations (32\%) being most frequent in probands confirmed with MODY. There was considerable regional variation in proband referral rates (from $<20$ per million in Wales and Northern Ireland to $>50$ per million for South West England and Scotland) and patients diagnosed with MODY (5.3 per million in Northern Ireland, 48.9 per million in South West England). Referral rates and confirmed cases were highly correlated
\end{abstract}

Electronic supplementary material The online version of this article (doi:10.1007/s00125-010-1799-4) contains supplementary material, which is available to authorised users.

B. M. Shields $\cdot$ S. Hicks $\cdot$ M. H. Shepherd $\cdot$ A. T. Hattersley $\cdot$

S. Ellard $(\square)$

Peninsula Medical School, University of Exeter,

Exeter, UK

e-mail: Sian.Ellard@rdeft.nhs.uk

K. Colclough $\cdot$ S. Ellard

Molecular Genetics Laboratory, Old Pathology Building,

Royal Devon and Exeter NHS Foundation Trust,

Barrack Road,

Exeter EX2 5DW, UK $(r=0.96, p<0.0001)$. The minimum prevalence of MODY was estimated to be 108 cases per million.

Conclusions/interpretation Assuming this minimal prevalence throughout the UK then $>80 \%$ of MODY is not diagnosed by molecular testing. The marked regional variation in the prevalence of confirmed MODY directly results from differences in referral rates. This could reflect variation in awareness of MODY or unequal access to genetic testing. Increased referral for diagnostic testing is required if the majority of MODY patients are to have the genetic diagnosis necessary for optimal treatment.

Keywords GCK · Genetic testing $\cdot$ HNF1A $\cdot$ MODY Prevalence. UK

\section{Introduction}

Maturity-onset diabetes of the young is a dominantly inherited form of non-insulin dependent diabetes that is typically diagnosed before 25 years of age and was first recognised by Tattersall in 1974 [1]. Today the term MODY is used to describe a group of clinically heterogeneous forms of beta cell dysfunction that are defined at the molecular genetics level by mutations in different genes.

The GCK gene encodes the pancreatic glucose sensor, glucokinase, a rate-limiting enzyme that catalyses the phosphorylation of glucose. Heterozygous loss-of-function mutations result in mild, fasting hyperglycaemia throughout life. Pharmacological treatment is rarely required and does not appear to alter glycaemic control (O. Gill-Carey, B.M. Shields, K. Colclough, S. Ellard and A.T. Hattersley, unpublished data). Mutations in the transcription factors 
encoded by $H N F 1 A$ and $H N F 4 A$ cause a progressive insulin secretory defect that shows sensitivity to low-dose sulfonylureas. Many cases of $H N F 1 A / 4 A$ MODY are misdiagnosed as type 1 or type 2 diabetes, but discovery of a mutation enables correct diagnosis, with subsequent alterations in treatment and improved glycaemic control in the majority of patients [2]. A genetic diagnosis also allows accurate estimation of diabetes risk for asymptomatic relatives.

$G C K$ and $H N F 1 A$ mutations are the most frequent cause of MODY in all populations studied [3]. The ratio of $G C K$ : $H N F 1 A$ mutations varies between countries due to different recruitment strategies for genetic testing; blood glucose screening in young, asymptomatic individuals will identify a higher proportion of $G C K$ mutations[4].

Mutations in the HNF1B, NEURODI, PDXI and INS genes are rare causes of MODY [5]. $H N F 1 B$ gene mutations were first described in a family with MODY, but the most common phenotype is renal cysts and diabetes (RCAD syndrome).

Based on clinical criteria, the prevalence of MODY in Germany was estimated at $0.14 \%$ or $1.8 \%$ of diabetes cases from studies of 40,927 and 2,064 diabetic patients, respectively [6, 7]. This corresponds to between 70 and 900 cases per million of the population. More recent data from a German/Austrian registry of 40,757 patients diagnosed with diabetes $<20$ years identified $0.83 \%$ MODY cases defined by clinical criteria and $0.65 \%$ confirmed by genetic testing [8]. The HUNT2 study tested 1,850 Norwegian diabetic patients for three $H N F 1 A$ mutations and found a minimum prevalence of $0.4 \%$ [9]. The prevalence of MODY in other populations has not been determined.

Genetic testing for MODY has been possible since identification of the major genetic causes in the 1990s. In this study we examined the referral patterns in the UK for genetic testing for MODY. The minimum prevalence was estimated from confirmed genetic diagnoses and the distribution of MODY subtypes was compared with that seen in other parts of Europe.

\section{Methods}

The Molecular Genetics Laboratory at the Royal Devon and Exeter Hospital is the UK referral centre for diagnostic testing of monogenic diabetes. Details of all patients referred for testing on a research or service basis are recorded on an in-house database.

All UK patients with diabetes diagnosed over the age of 1 year and referred for MODY testing between 1 January 1996 and 28 February 2009 were identified from this database. Genetic testing was conducted according to the clinical phenotype and included $G C K, H N F 1 A, H N F 4 A$,
HNF1B, INS, PDX1 and NEURODI sequence analysis. Dosage testing for partial/full gene deletions was included for the $G C K, H N F 1 A, H N F 4 A$ and $H N F 1 B$ genes. Guidelines for genetic testing in MODY were published in 2008 [10].

Patients were assigned to regions according to their home address, if available, or referring clinician. These were defined by the UK Government Office Regions (www.statistics.gov.uk/geography/gor.asp, accessed 6 May 2010). Population estimates for these regions were obtained from 2001 census data (www.statistics.gov.uk/census2001/ population_data.asp, accessed 6 May 2010).

For analysis purposes we defined two distinct groups: (1) proband referrals, the first member of a family referred for genetic testing; and (2) MODY cases, which includes all patients (probands and relatives) with a genetic diagnosis of their diabetes. We also included the diabetic relatives of non-diabetic probands with $H N F 1 B$ mutations within the MODY cases. These non-diabetic probands were predominantly paediatric cases referred by nephrologists.

\section{Results}

Proband referrals for genetic testing A total of 2,072 UK probands with diabetes were referred for MODY genetic testing between 1996 and 2009. Mutations were identified in $564(27 \%)$, confirming a diagnosis of MODY. The number of referrals per year has increased from 65 in 1996 to 351 in 2008 (Electronic supplementary material [ESM] Fig. 1).

Testing of relatives A total of 1,280 relatives from 336 families were tested (ESM Fig. 2). These included 745 family members with diabetes, of whom 613 had inherited the familial mutation. Of the 535 relatives not known to have diabetes, 71 had inherited the mutation and have a high lifetime risk of developing MODY.

Genetic causes In the 564 diabetic probands with confirmed MODY, $H N F 1 A$ mutations were most common (52\%), followed by GCK (32\%), HNF4A (10\%) and HNF1B $(6 \%)$. The remainder had mutations in INS or NEURODI $(<1 \%)$.

Geographical variation in referral rates and confirmed cases Referral rates for diabetic probands ranged between the constituent countries from 17.8 per million of the population in Northern Ireland to 57.7 in Scotland (Table 1). The English regions showed even greater variation, ranging from 20.6 in the East, to 105.9 in the South West. These data are illustrated in Fig. 1a. The lowest referral rates were 
Table 1 Regional variation in referrals for genetic testing for MODY and diabetic cases with a confirmed diagnosis of MODY

\begin{tabular}{|c|c|c|c|c|c|c|c|c|}
\hline \multicolumn{2}{|l|}{ Geographical factors } & \multicolumn{4}{|l|}{ Probands } & \multirow{2}{*}{$\begin{array}{l}\text { Relatives } \\
\text { Diabetic relatives } \\
\text { with mutation }(n)\end{array}$} & \multicolumn{2}{|l|}{$\operatorname{Cases}^{\mathrm{a}}$} \\
\hline $\begin{array}{l}\text { Country/ } \\
\text { region }\end{array}$ & Population & $\begin{array}{l}\text { Referrals } \\
(n)\end{array}$ & $\begin{array}{l}\text { Referrals } \\
\text { per million }\end{array}$ & $\begin{array}{l}\text { With } \\
\text { mutation }(n)\end{array}$ & $\begin{array}{l}\text { Pick-up } \\
\text { rate }(\%)^{b}\end{array}$ & & $\begin{array}{l}\text { Total } \\
\text { cases }(n)\end{array}$ & $\begin{array}{l}\text { Total cases } \\
\text { per million }\end{array}$ \\
\hline Scotland & $5,062,011$ & 292 & 57.7 & 74 & 25.3 & 70 & 144 & 28.4 \\
\hline Wales & $2,903,085$ & 38 & 13.1 & 14 & 36.8 & 22 & 36 & 12.4 \\
\hline Northern Ireland & $1,685,267$ & 30 & 17.8 & 7 & 23.3 & 2 & 9 & 5.3 \\
\hline England & $49,138,831$ & 1,712 & 34.8 & 469 & 27.4 & 519 & 988 & 20.1 \\
\hline \multicolumn{9}{|l|}{ English regions } \\
\hline East & $5,388,154$ & 111 & 20.6 & 36 & 32.4 & 44 & 80 & 14.8 \\
\hline South East & $8,000,550$ & 219 & 27.4 & 71 & 32.4 & 71 & 142 & 17.7 \\
\hline South West & $4,928,458$ & 522 & 105.9 & 107 & 20.5 & 134 & 241 & 48.9 \\
\hline London & $7,172,036$ & 182 & 25.4 & 39 & 21.4 & 20 & 59 & 8.2 \\
\hline West Midlands & $5,267,337$ & 167 & 31.7 & 45 & 26.9 & 62 & 107 & 20.3 \\
\hline East Midlands & $4,172,179$ & 109 & 26.1 & 34 & 31.2 & 34 & 68 & 16.3 \\
\hline Yorkshire/Humber & $4,964,838$ & 128 & 25.8 & 46 & 35.9 & 46 & 92 & 18.5 \\
\hline North East & $2,515,479$ & 94 & 37.4 & 34 & 36.2 & 19 & 53 & 21.1 \\
\hline North West & $6,729,800$ & 175 & 26.0 & 55 & 31.4 & 53 & 108 & 16.0 \\
\hline Unknown & & 5 & & 2 & & 36 & 38 & \\
\hline UK total & $58,789,194$ & 2,072 & $35.2^{\mathrm{c}}$ & 564 & $27.2^{\mathrm{c}}$ & 613 & 1,177 & $20.0^{\mathrm{c}}$ \\
\hline
\end{tabular}

Proband referral rates and confirmed cases were calculated per million of regional population

${ }^{\mathrm{a}}$ Probands + relatives

${ }^{\mathrm{b}}$ Minimum \% pick-up rate, based on number of probands with mutations identified per all proband referrals for region specified

${ }^{\mathrm{c}}$ Values averaged

observed in Northern Ireland and Wales $(<20)$, and the highest in Scotland and the South West of England ( $>50$ proband referrals per million of population).

Similar variation was seen for the number of MODY cases confirmed by genetic testing (Table 1, Fig. 1b). Again, the South West and Scotland had the highest 'scores', with 48.9 and 28.4 cases of MODY per million, respectively, while Northern Ireland was lowest (5.3 per million). Referrals and confirmed cases across the UK regions were highly correlated $(r=0.96, p<0.00001)$.

Pick-up rate according to clinical criteria Information on age at diagnosis, family history and current treatment was available for $1,492 / 2,072$ probands $(72 \%)$. The minimum pick-up rate for those patients meeting clinical criteria for MODY (age at diagnosis less than 25 years, at least one parent affected, not insulin treated) was $51 \%$, compared with $23 \%$ for patients who did not (ESM Table 1).

Estimated prevalence of MODY in the UK To estimate the minimum prevalence of MODY, we examined the data for the two places with the highest number of confirmed cases, Exeter and Edinburgh Lothians. Within the EX1-EX4 central postcode area of Exeter, 12 patients from a population of 111,076 had a genetic diagnosis of MODY (108 cases per million). The Lothians area has a population of 778,367 with 53 confirmed MODY cases (68 cases per million). These figures suggest a minimum UK prevalence of 6,351 or 4,003 patients respectively.

\section{Discussion}

This is the first study to calculate the minimum population prevalence of MODY from genetic diagnoses, reporting, in addition, the largest number of confirmed MODY cases $(n=1,177)$ from a single country. The main limitation of our study is that the data are based on referrals for genetic testing rather than on a systematic survey of a static population. The minimum prevalence of 108 cases per million population is higher than the first report from Germany based on clinical criteria [6], but much lower than the later study [7]. More recent data from diabetes registers in Germany/Austria and Norway showed a genetic diagnosis of MODY frequency of $0.65 \%$ (263 patients) and $0.4 \%$ (eight patients) $[8,9]$. 
Fig. 1 Regional variation in (a) proband referrals for genetic testing of MODY and (b) cases with a genetic diagnosis of MODY. Both are shown per million of population

The number of confirmed MODY cases shows a more than fivefold variation between the constituent UK countries (from 5.3 cases per million of the population in Northern Ireland to 28.4 in Scotland) and across the English regions (from 8.2 in London to 48.9 in the South West). The high correlation with referral rates $(r=0.96)$ suggests that this variation results from unequal use of genetic testing.

There are many possible reasons for this variability in use of genetic testing for MODY. These include knowledge about MODY, the financial cost of testing and ethnic differences in prevalence or referral rates. For example, the Indian population has a higher prevalence of diabetes with earlier onset of type 2 diabetes and lower BMI, so there is more overlap with the MODY phenotype than in European Whites. A previous UK survey found that Asian MODY cases were under-represented, comprising only $0.5 \%$ of referrals for MODY testing, but $4 \%$ of the population [11].

MODY is a relatively rare condition and awareness of the clinical phenotype and availability of testing are likely to vary. The South West had the highest referral rates and number of diagnosed cases, reflecting the strong research interest in MODY in this area. The UK Genetic Diabetes Nurse project has provided training for 26 diabetes specialist nurses [12], but none of these were from Northern Ireland or Wales, the countries with the lowest numbers of confirmed MODY cases. Genetic testing is expensive compared with routine biochemical and immunological tests used in the diagnosis and management of diabetes. Central funding for genetic testing is provided only in Scotland, so it is, perhaps, no coincidence that this is the constituent country with the highest referral rate.

The distribution of MODY subtypes reflects the referral pattern from hospital clinics rather than general practice or paediatric screening, with $H N F 1 A / 4 A$ mutations (62\%) nearly twice as frequent as GCK (32\%). HNF1A MODY is also the most common subtype in the Netherlands and Denmark [3]. In contrast, GCK mutations predominate in Spain, Italy, France, Germany and the Czech Republic where more paediatric cases are tested $[3,8]$.

What is the prevalence of MODY? If it accounts for $1 \%$ of diabetes, then approximately 26,000 cases are predicted in the UK. In this study, we report a minimum prevalence of $\sim 6,000$ cases, but the exact prevalence will remain unknown until population-based studies are performed. However, with the genetic diagnosis of only 1,177 UK cases to date, our data highlight that the majority of cases remain misdiagnosed or undiagnosed. This means that thousands of patients are not receiving the most appropriate
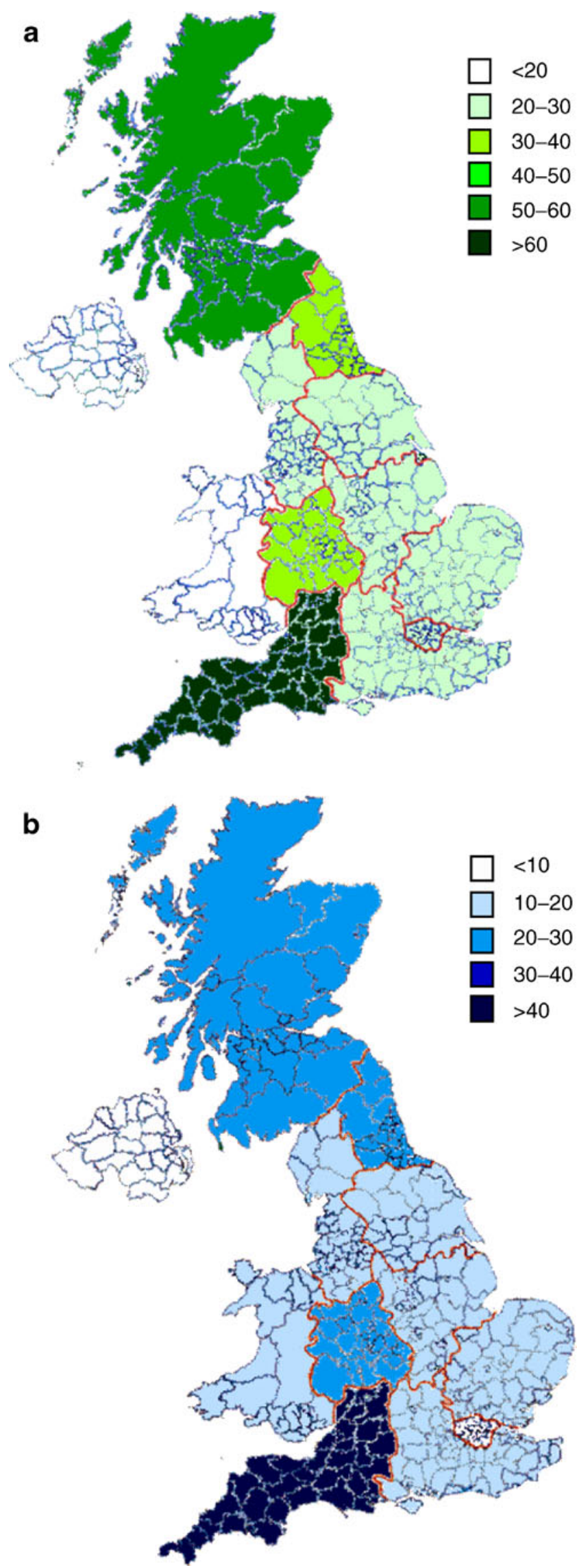
treatment and their unaffected relatives are unaware of their increased risk of diabetes.

Acknowledgements Diabetes UK supported the MODY genetic testing from 1996 until 2000. We would like to thank all the Genetics Diabetes Nurses, referring clinicians and the Exeter Molecular Genetics Laboratory staff for their contribution to this work. J. Little provided additional data for the Lothians area referrals. B.M. Shields, M. H. Shepherd, A.T. Hattersley and S. Ellard are employed as core members of the Peninsula NIHR Clinical Research Facility.

Duality of interest The authors declare that there is no duality of interest associated with this manuscript.

\section{References}

1. Tattersall RB (1974) Mild familial diabetes with dominant inheritance. Q J Med 43:339-357

2. Shepherd M, Shields B, Ellard S, Rubio-Cabezas O, Hattersley AT (2009) A genetic diagnosis of HNF1A diabetes alters treatment and improves glycaemic control in the majority of insulin-treated patients. Diabet Med 26:437-441

3. Estalella I, Rica I, Perez de Nanclares G et al (2007) Mutations in GCK and HNF-1alpha explain the majority of cases with clinical diagnosis of MODY in Spain. Clin Endocrinol (Oxf) 67:538-546
4. Chevre JC, Hani EH, Boutin P et al (1998) Mutation screening in 18 Caucasian families suggest the existence of other MODY genes. Diabetologia 41:1017-1023

5. Murphy R, Ellard S, Hattersley AT (2008) Clinical implications of a molecular genetic classification of monogenic beta-cell diabetes. Nat Clin Pract Endocrinol Metab 4:200-213

6. Panzram G, Adolph W (1981) Heterogeneity of maturity onset diabetes at young age (MODY). Lancet 2:986

7. Ledermann HM (1995) Maturity-onset diabetes of the young (MODY) at least ten times more common in Europe than previously assumed? Diabetologia 38:1482

8. Schober E, Rami B, Grabert M et al (2009) Phenotypical aspects of maturity-onset diabetes of the young (MODY diabetes) in comparison with type 2 diabetes mellitus (T2DM) in children and adolescents: experience from a large multicentre database. Diabet Med 26:466-473

9. Eide SA, Raeder H, Johansson S et al (2008) Prevalence of HNF1A (MODY3) mutations in a Norwegian population (the HUNT2 Study). Diabet Med 25:775-781

10. Ellard S, Bellanne-Chantelot C, Hattersley AT (2008) Best practice guidelines for the molecular genetic diagnosis of maturity-onset diabetes of the young. Diabetologia 51:546-553

11. Porter JR, Rangasami JJ, Ellard S et al (2006) Asian MODY: are we missing an important diagnosis? Diabet Med 23:12571260

12. Shepherd M, Hattersley AT, Ellard S (2005) Integration of the MODY link nurse project: 20 month evaluation. J Diabetes Nurs 9:47-52 